\title{
Does Coronal Knee and Ankle Alignment Affect Recurrence of the Varus Deformity after High Tibial Osteotomy?
}

\author{
O-Sung Lee, $\mathrm{MD}^{1}$, Seung Hoon Lee, $\mathrm{MD}^{2}$, and Yong Seuk Lee, $\mathrm{MD}, \mathrm{PhD}^{3}$ \\ ${ }^{1}$ Department of Orthopaedic Surgery, Mediplex Sejong Hospital, Incheon; ${ }^{2}$ Department of Orthopaedic Surgery, Incheon Metropolitan City Medical Center, Incheon; \\ ${ }^{3}$ Department of Orthopaedic Surgery, Seoul National University Bundang Hospital, Seoul National University College of Medicine, Seongnam, Korea
}

\begin{abstract}
Purpose: The purpose of this study was to evaluate changes in the coronal alignment of the knee and ankle joints after open wedge high tibial osteotomy (OWHTO) to determine factors related to the recurrence (R) of the varus deformity by serial analysis.

Materials and Methods: Sixty-four OWHTOs were enrolled in this study. The weight bearing line (WBL) ratio, joint line convergence angle (JLCA), knee joint inclination, mechanical axis-tibial plateau angle, talar inclination (TI), and distal tibia articular angle (DTAA) were serially assessed. Serial correlation analysis between all parameters was performed. Patients were divided into R group and no recurrence (NR) group according to the WBL ratio $(55 \%)$ at postoperative one year.

Results: The preoperative WBL ratio showed significantly negative correlation with serial changes of JLCA, TI, and DTAA (p<0.05). The JLCA, TI, and DTAA as well as WBL ratio showed a significantly larger degree of varus alignment in the R group than in NR group at postoperative 6 weeks and 1 year after OWHTO $(\mathrm{p}<0.05)$.

Conclusions: Sufficient correction of the WBL and restoration of the JLCA during OWHTO are essential to prevention of the R of varus deformity after the surgery because they are the only modifiable factors during surgery.

Level of Evidence: IV, Case series
\end{abstract}

Keywords: Knee, Osteoarthritis, Varus deformity, Osteotomy, Recurrence

\section{Introduction}

Open wedge high tibial osteotomy (OWHTO) is generally accepted as one of the most useful surgical procedures for treating medial compartment osteoarthritis of the knee ${ }^{1-77}$. By changing

Received May 24, 2018; Revised (1st) August 30, 2018;

(2nd) September 18, 2018; Accepted September 21, 2018

Correspondence to: Yong Seuk Lee, MD, PhD

Department of Orthopaedic Surgery, Seoul National University Bundang Hospital, Seoul National University College of Medicine, 182 Gumi-ro

173beon-gil, Bundang-gu, Seongnam 13620, Korea

Tel: +82-31-787-7199, Fax: +82-31-787-4056

E-mail: smcos1@snu.ac.kr

Source of funding: This research was supported by Basic Science

Research Program through the National Research Foundation of Korea

(NRF) funded by the Ministry of Education (2014R1A1A2053356).

This is an Open Access article distributed under the terms of the Creative Commons Attribution Non-Commercial License (http://creativecommons.org/licenses/by-nc/4.0/) which permits unrestricted non-commercial use, distribution, and reproduction in any medium, provided the original work is properly cited. the alignment of the knee joint from varus to valgus, an excessive medial load is shifted to the lateral compartment, which results in less degenerative changes ${ }^{8}$. Furthermore, the OWHTO could affect not only the alignment of the knee joint but also the alignment of the ankle joint ${ }^{8)}$.

Each deformity of the knee and ankle joint plays a role in determining the overall alignment of the lower extremity. It seems likely that some compensatory changes occur at each joint in response to deformity of the other. Recognition of the compensatory relationship between these joints and precise information about where they occur would provide guidance for knee deformity correction".

However, one major issue after OWHTO is the recurrence (R) of varus deformity despite proper surgical correction for knee alignment within the generally acceptable range. Therefore, concerns have arisen about various factors that might affect the results of operation, and the coronal alignment of the knee joint has been evaluated to investigate related factors ${ }^{4,10)}$. However, little is 
known about the interaction between the factors and changes in the mechanical axis of the lower extremity. In addition, we still do not know how those factors affect $\mathrm{R}$ of the varus deformity ${ }^{4,10,11)}$.

The purpose of this study was to evaluate changes in the factors associated with the coronal alignment of the knee and ankle joints after OWHTO to determine which factors are related to the $\mathrm{R}$ of the varus deformity by serial assessment. The hypotheses of this study were (1) serial changes in the parameters representing the coronal alignment of each joint would be inter-related and (2) there would be some factors affecting the R after OWHTO.

\section{Materials and Methods}

\section{Demographics}

From March 2014 to December 2014, a total of 60 consecutive patients (64 consecutive lower limbs) that underwent biplanar OWHTO with a mean age of $57 \pm 5.73$ years were enrolled in this study. The OWHTO was performed in all patients under the diagnosis of varus medial compartment osteoarthritis. The inclusion criteria for this study were selected from those described in the literature: (1) primary degenerative osteoarthritis (not inflammatory arthritis), (2) radiographic evidence of isolated medial compartment osteoarthritis (Kellgren-Lawrence grade III or IV) in the knee joint, (3) concurrent varus deformity of the lower limb, and (4) failure of conservative treatments. Surgical indications included relatively active patients aged less than 70 years. Patients with mild patellofemoral arthritis were also included ${ }^{12)}$. The exclusion criteria were as follows: (1) history of double osteotomy, (2) HTO for correction of the different pattern of the deformity, (3) secondary osteoarthritis such as post-traumatic arthritis and sequelae of septic arthritis, (4) cases with the collapsed medial proximal tibial angle (MPTA) within postoperative 1 year due to a metal failure or a lateral hinge fracture. The Institutional
Review Board approval was obtained before commencement of the study.

\section{Surgical Technique}

An approximately $5 \mathrm{~cm}$ incision is made longitudinally at the $1 \mathrm{~cm}$ anterior portion of the posterior crest of the tibia. This incision is more posterior than the usual incision because it allows for easy insertion of a releaser and a protector. The interval behind the patellar tendon is now freed, and the insertion area of the tendon is protected using a retractor. Then, the superior border of the pes anserinus is incised, the medial collateral ligament is mobilized from the tibia, and a release is performed by insertion of a periosteal elevator.

Release behind the posteromedial cortex of the tibia is typically done using gauge packing, which enables access to the more than half of the posterior cortex of the tibia. After removal of the gauge, the releaser is inserted through this interval and further release is performed by pushing the releaser until contact is made with the posterior cortex. If the tip of the releaser reaches the fibular head area, the protector is inserted at the interval between the posterior cortex and the releaser, and the releaser is removed.

With the help of anteroposterior (AP) C-arm images, the tip of the protector is hooked to the target portion of the hinge located at the lateral cortex of the proximal tibia. Then, the cutting block is attached to the protector and pushed to the posteromedial cortex of the tibia. If contact is made, the cutting block is tightened to the protector and guiding pins are inserted at the four holes of the cutting block ${ }^{10)}$. Finally, sawing is performed; the main goal of this procedure is sawing of the posterior cortex. After removal of the protective cutting complex, C-arm images are checked. Finally, anterior retro-tubercle osteotomy is performed with distraction at the most posterior gap. The amount of distraction or control of tibial slope is adjusted according to preoperative
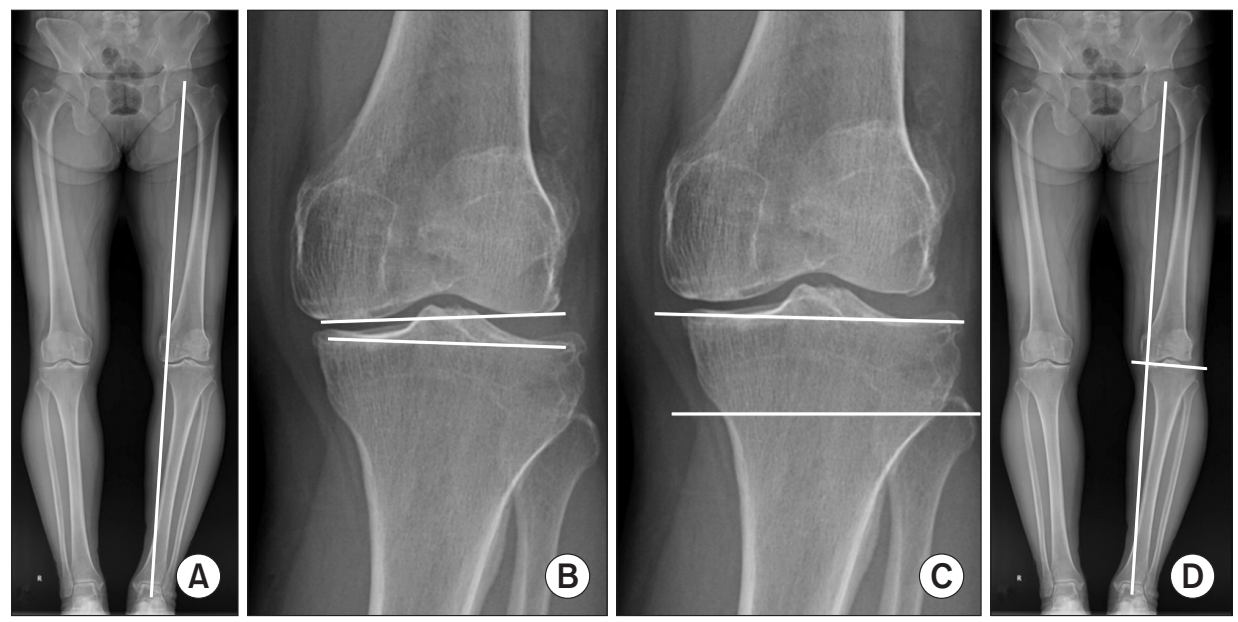

Fig. 1. Weight bearing line (WBL), joint line convergence angle (JLCA), knee joint inclination (KI), and mechanical axis-tibial plateau (MA-TP) angle. (A) The WBL is drawn from the center of the femoral head to the center of the talus. The WBL ratio is calculated in the tibial plateau from the medial edge. (B) The JLCA is the angle formed between two tangential lines at the distal femoral and proximal tibial articular surfaces. (C) The KI is the angle between the horizontal line at the proximal tibial articular surfaces and the line parallel to the tibial plateau. (D) The MA-TP angle is the angle between the mechanical axis and the tangential line at the tibial plateau. 
planning at the most posterior gap ${ }^{4}$. Usually, the target point of correction is around $62.5 \%$ (range, $55 \%$ to $65 \%$ ) of the weight bearing line (WBL). In our patients, if the medial compartment showed severe degeneration, the target point was shifted toward a larger correction of approximately $65 \%$.

\section{Evaluations}

INFINITT ver. 5.0.9.2 (INFINITT, Seoul, Korea) was used for all radiographic measurements. Standing AP weight bearing whole leg radiographs taken immediately after surgery and postoperative 3 months, 6 months, and 1 year were used for the serial assessment. The WBL was serially evaluated and its correlation with other factors was investigated.

$\mathrm{R}$ of the varus deformity was defined as the WBL ratio less than $55 \%$ in whole leg radiographs at 1 year after surgery because our target point of WBL ratio was adjusted 55\%-65\% (around 62.5\%) according to the status of the medial compartment of the knee joint. To evaluate related factors, patients were divided into the $\mathrm{R}$ group (WBL $<55 \%)$ or no recurrence (NR) group (WBL $\geq 55 \%)$ according to the postoperative 1-year WBL ratio. Therefore, we defined the $\mathrm{R}$ group as patients having a WBL ratio of less than $55 \%$ during postoperative 1 year follow-up. Parameters that showed statistically significant correlation with the WBL were compared between the two groups.

In the knee joint, preoperative and postoperative WBL, joint line convergence angle (JLCA), knee joint inclination (KI), and mechanical axis-tibial plateau (MA-TP) angle were evaluated. The WBL was drawn from the center of the femoral head to the center of the superior articular surface of the talus (Fig. 1). To calculate the WBL ratio, the denominator was the width of the tibia, measured using a ruler, and the numerator was the tibial intersection of the WBL (with the medial tibial edge at $0 \%$ and the lateral tibial edge at $100 \%)$.

To evaluate soft tissue laxity on the coronal plane, the JLCA
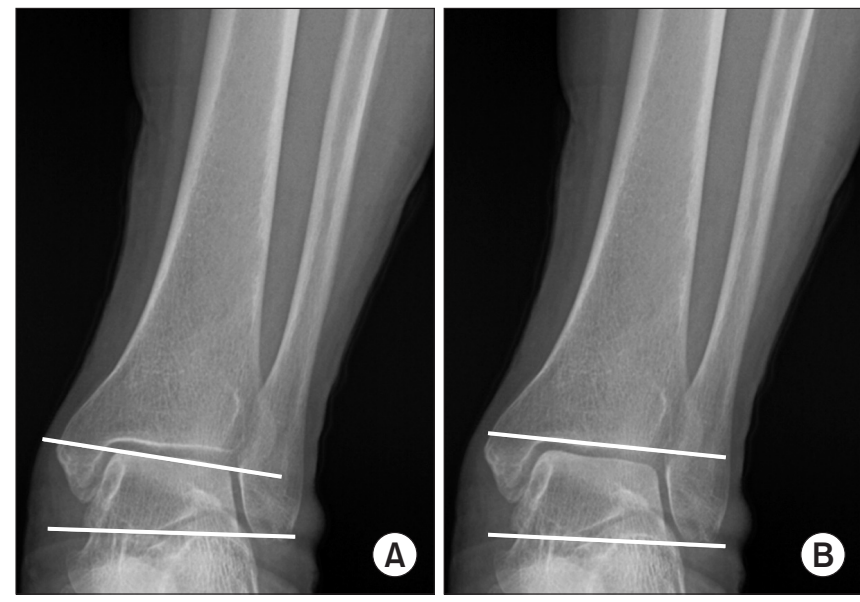

Fig. 2. Talar inclination (TI) and distal tibia articular angle (DTAA). (A) The TI is the angle between the horizontal line and the tangential line at the talar dome. (B) The DTAA is the angle between the horizontal line and the tangential line at the distal tibial plafond.

Table 1. Correlation Analysis between Weight Bearing Line and Other Parameters

\begin{tabular}{|c|c|c|c|c|c|}
\hline Parameter & Preoperative & Immediate postop & Postop 3 months & Postop 6 months & Postop 1 year \\
\hline Joint line convergence angle $\left({ }^{\circ}\right)$ & $2.17 \pm 1.48$ & $1.91 \pm 1.76$ & $1.93 \pm 1.83$ & $1.94 \pm 1.74$ & $1.91 \pm 1.95$ \\
\hline PCC & -0.49 & -0.33 & -0.35 & -0.34 & -0.44 \\
\hline Significant probability & 0.01 & 0.01 & 0.01 & 0.01 & 0.01 \\
\hline Knee joint inclination angle $\left(^{\circ}\right)$ & $1.24 \pm 1.85$ & $2.65 \pm 2.71$ & $3.03 \pm 2.83$ & $3.34 \pm 2.77$ & $3.46 \pm 2.73$ \\
\hline PCC & -0.22 & 0.21 & 0.06 & 0.18 & 0.21 \\
\hline Significant probability & 0.01 & 0.12 & 0.65 & 0.21 & 0.13 \\
\hline Mechanical axis-tibial plateau angle $\left(^{\circ}\right)$ & $88.67 \pm 2.61$ & $91.26 \pm 2.17$ & $90.96 \pm 2.54$ & $91.14 \pm 2.45$ & $91.17 \pm 2.18$ \\
\hline PCC & -0.06 & 0.27 & 0.31 & 0.04 & 0.15 \\
\hline Significant probability & 0.68 & 0.04 & 0.02 & 0.79 & 0.28 \\
\hline Talar inclination $\left(^{\circ}\right)$ & $5.91 \pm 4.25$ & $-1.44 \pm 3.89$ & $0.64 \pm 4.39$ & $0.81 \pm 4.36$ & $1.11 \pm 4.27$ \\
\hline PCC & -0.43 & -0.26 & -0.4 & -0.61 & -0.52 \\
\hline Significant probability & 0.01 & 0.05 & 0.01 & 0.01 & 0.01 \\
\hline Distal tibia articular angle $\left(^{\circ}\right)$ & $4.81 \pm 3.59$ & $-1.68 \pm 3.42$ & $-0.75 \pm 3.51$ & $0.31 \pm 3.64$ & $0.67 \pm 3.34$ \\
\hline PCC & -0.35 & -0.28 & -0.45 & -0.61 & -0.52 \\
\hline Significant probability & 0.01 & 0.04 & 0.01 & 0.01 & 0.01 \\
\hline
\end{tabular}

Values are presented as mean \pm standard deviation.

Postop: postoperative, PCC: Pearson correlation coefficient. 
was measured as the angle between the line connecting the distal femur and the proximal tibial articular surfaces (Fig. 1B). If the apex of the JLCA was medial, it was recorded as positive (+) and denoted as varus; if it was lateral, it was recorded as negative $(-)$ and denoted as valgus ${ }^{10)}$. The KI was measured as the angle between a line horizontal to the ground and a line tangent to the tibial plateau, with the varus position considered a positive $(+)$ angle (Fig. 1C). The MA-TP angle was measured as the angle between the mechanical axis and a line tangent to the tibia plateau (Fig. 1D). The varus position was considered a positive (+) angle. In the ankle joint, the talar inclination (TI) angle and distal tibia articular angle (DTAA) were evaluated. The TI was measured as the angle between the superior surface of the talar dome and the horizontal line (Fig. 2A). The DTAA was measured as the angle between the surface of the tibial plafond and the horizontal line, with the varus position considered a positive (+) angle (Fig. 2B).

In order to evaluate related factors, serial correlation analysis between all parameters was performed. The affecting factors, which were inter-related, were compared between the R and NR groups serially.

\section{Statistical Analysis}

SPSS ver. 18.0 (SPSS Inc., Chicago, IL, USA) was used for all analyses. A p-value $<0.05$ was considered significant. To evaluate whether correlations exist between the WBL and other parameters in the serial assessment, Pearson's correlation analysis was performed. To compare between the R and NR groups, Student $t$-test or Mann-Whitney test was performed according to normality of data. Reliability of measurements was assessed by examining the intra-rater and inter-rater reliability using intraclass correlation coefficients (ICCs). Two orthopedic surgeons in the clinical fellowship program working in the orthopedic department performed the measurements twice at 2-week intervals.

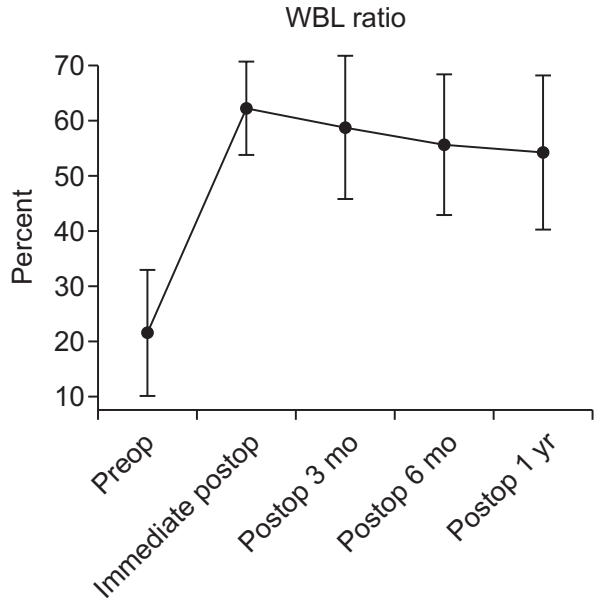

JLCA

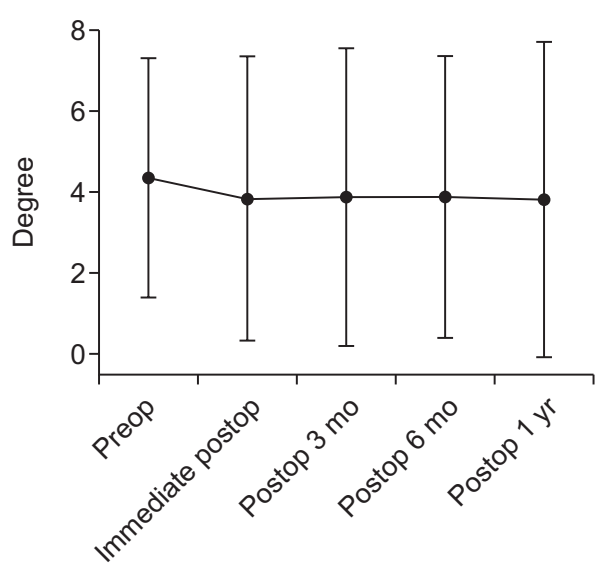

MA-TP

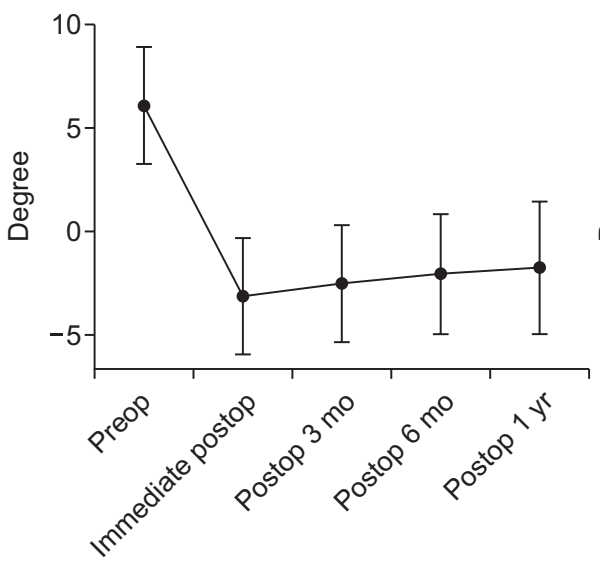

TI

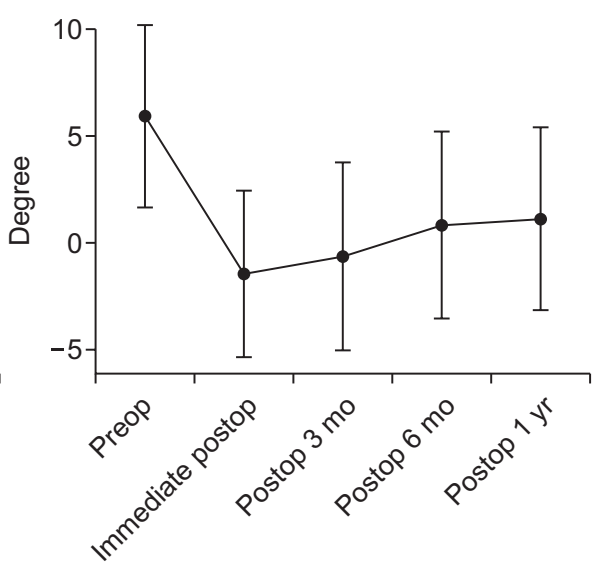

$\mathrm{KI}$

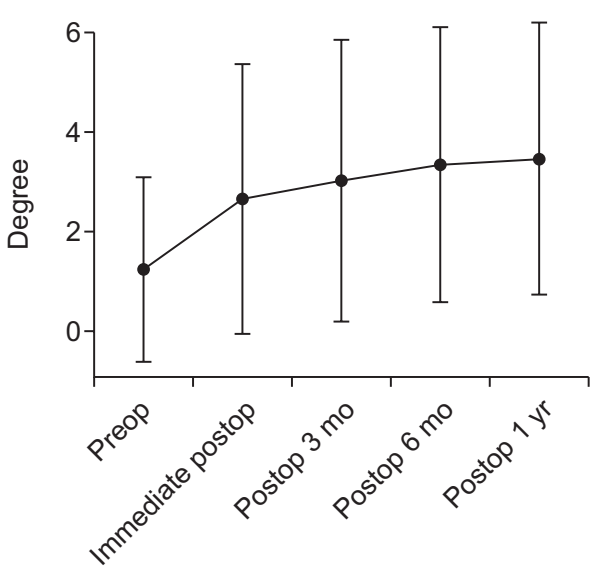

DTAA

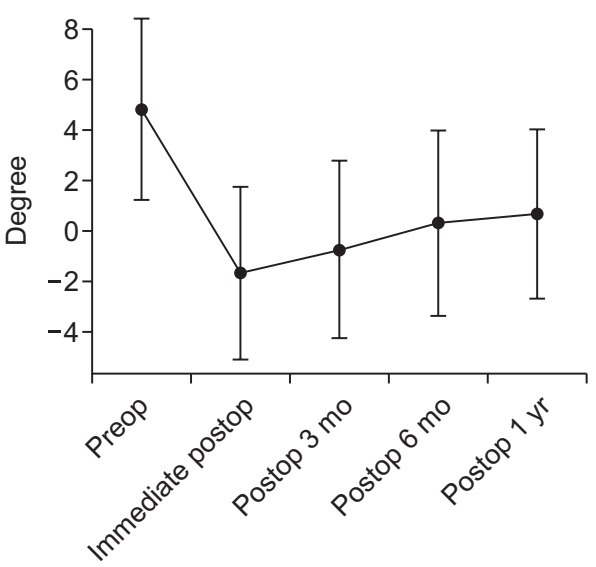

Fig. 3. Serial changes of each parameter. WBL: weight bearing line, MA-TP: mechanical axis-tibial plateau, KI: knee joint inclination, JLCA: joint line convergence angle, TI: talar inclination, DTAA: distal tibia articular angle, Preop: preoperative, Postop: postoperative. 
Results

Inter-observer and intra-observer reliability of the measurements were satisfactory, with a mean ICC value of 0.79 (range, 0.7 to 0.87 ) and 0.9 (range, 0.82 to 0.93 ), respectively. The WBL was serially changed from $21.59 \pm 11.36$ preoperatively to $62.16 \pm 8.39$ immediately postoperatively, to $58.63 \pm 12.92$ at 3 months postoperatively, to $55.59 \pm 12.72$ at 6 months postoperatively, and then to $54.14 \pm 13.87$ at 1 year postoperatively.

On the correlation analysis of all related factors, the preoperative WBL ratio was serially correlated with the preoperative and postoperative JLCA, TI angle, and DTAA continuously up to 1 year after surgery. The JLCA, TI angle, and DTAA showed statistically significantly negative correlations with the preoperative WBL at all times of assessment. However, the KI angle showed no statistically significant correlation with the preoperative WBL at any stages of the serial assessment. The MA-TP angle till 3 months after surgery was significantly correlated with the preoperative WBL; however, the correlation did not persist from 6 months after surgery (Table 1, Fig. 3).

At one year after OWHTO, the R group and NR group comprised 17 knees (26\%) and 47 knees (74\%), respectively. The
A
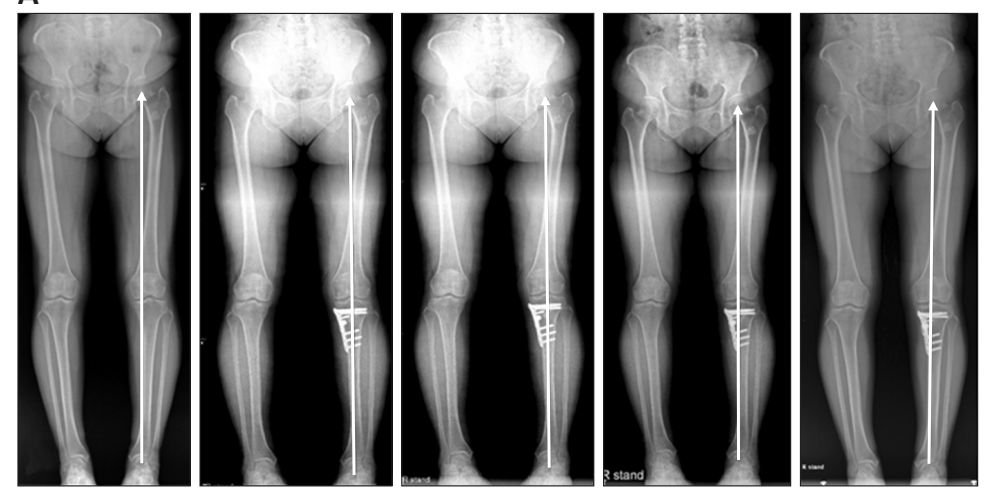

C
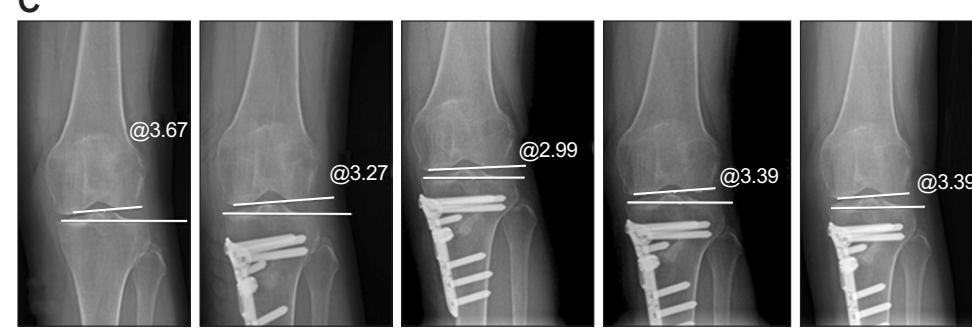

E
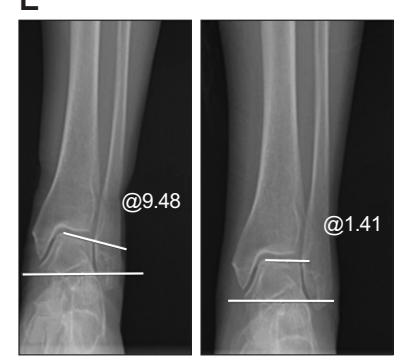
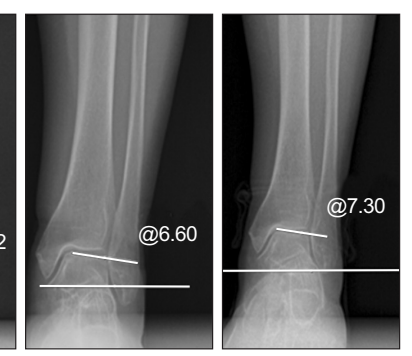

B

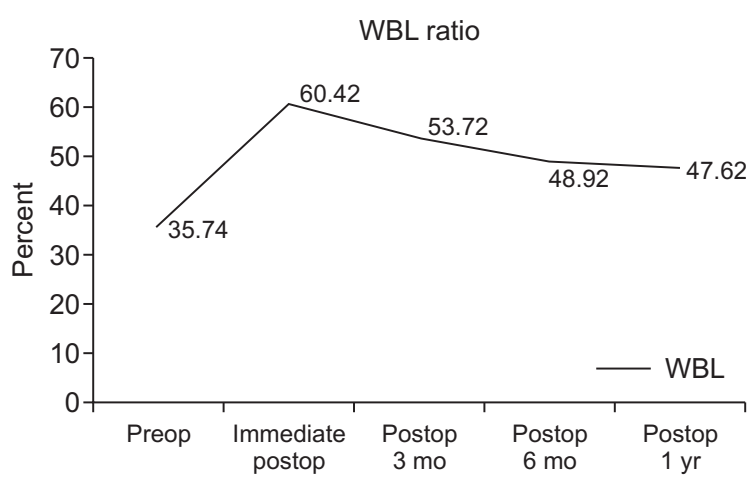

JLCA

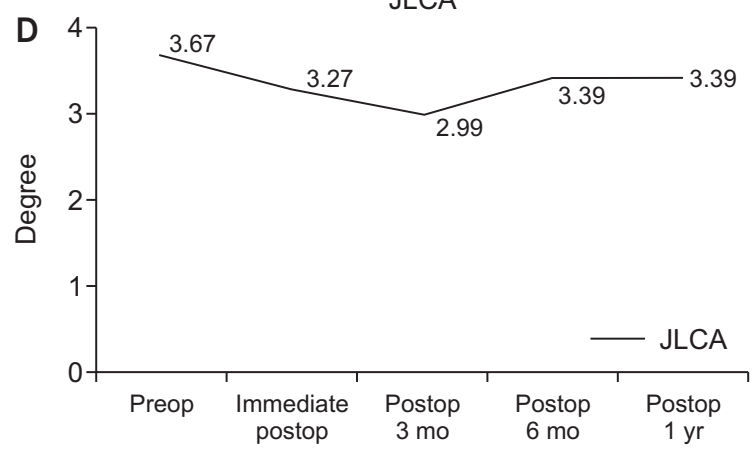

TI

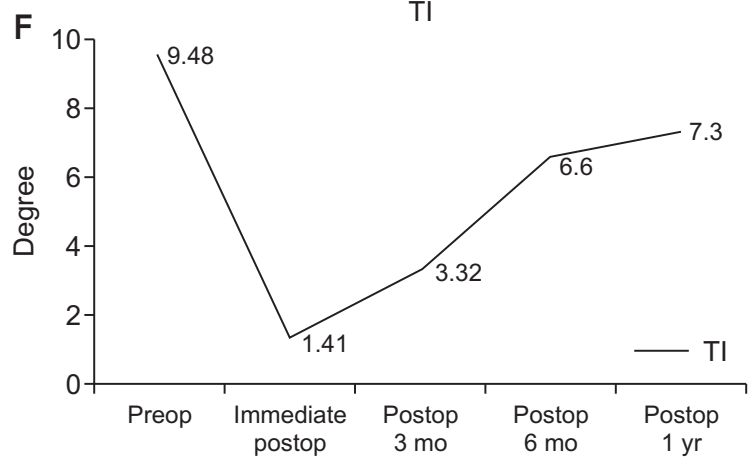

Fig. 4. Serial changes of each parameter in the no reccurence group. (A) Weight bearing line (WBL) ratio in the whole leg radiograph. (B) WBL ratio in the polygonal line graph. (C) Joint line convergence angle (JLCA) in the whole leg radiograph. (D) JLCA in the polygonal line graph. (E) Talar inclination (TI) in the whole leg radiograph. (F) TI in the polygonal line graph. Preop: preoperative, Postop: postoperative. 
WBL ratio of the $\mathrm{R}$ group and NR group was $56.83 \% \pm 9.40 \%$ (range, $51.8 \%$ to $62.2 \%$ ) and $64.50 \% \pm 6.65 \%$ (range, $61.8 \%$ to $73.9 \%)$ at postoperative 6 weeks $(\mathrm{p}=0.001)$ and $37.30 \% \pm 10.52 \%$ (range, $27.6 \%$ to $52.9 \%$ ) and $61.02 \% \pm 7.59 \%$ (range, $56.3 \%$ to $73.6 \%)$ at postoperative 1 year $(\mathrm{p}<0.001)$. The changes of the JLCA, TI angle, and DTAA, which showed statistically significant correlation with the preoperative WBL ratio, were compared between the R and NR groups (Figs. 4 and 5). The mean JLCA at postoperative 6 weeks and 1year showed a significantly larger varus alignment in the $\mathrm{R}$ group than in the NR group despite the similar degree of preoperative JLCA (postoperative 6 weeks, $2.91^{\circ} \pm 2.21^{\circ}$ vs. $1.60^{\circ} \pm 1.47^{\circ} ; \mathrm{p}=0.005$; postoperative 1 year, $3.29^{\circ} \pm 2.28^{\circ}$ vs. $1.32^{\circ} \pm 1.44^{\circ} ; \mathrm{p}<0.001$ ). The TI and DTAA at postoperative 6 weeks and 1year also showed significantly larger varus alignment in the $\mathrm{R}$ group than in the NR group. The TI of the R group and NR group was $1.04^{\circ} \pm 4.21^{\circ}$ and $-1.22^{\circ} \pm 3.81^{\circ}$ at postoperative 6 weeks $(\mathrm{p}=0.048)$ and $3.89^{\circ} \pm 4.94^{\circ}$ and $-0.33^{\circ} \pm 3.59^{\circ}$ at postoperative 1 year $(\mathrm{p}=0.001)$. The DTAA of the $\mathrm{R}$ group and NR group was $0.23^{\circ} \pm 2.77^{\circ}$ and $-1.58^{\circ} \pm 3.17^{\circ}$ at postoperative 6 weeks $(\mathrm{p}=0.043)$ and $3.00^{\circ} \pm 3.72^{\circ}$ and $-0.13^{\circ} \pm 3.19^{\circ}$ at postoperative 1 year $(\mathrm{p}=0.002)$ (Table 2$)$.
A

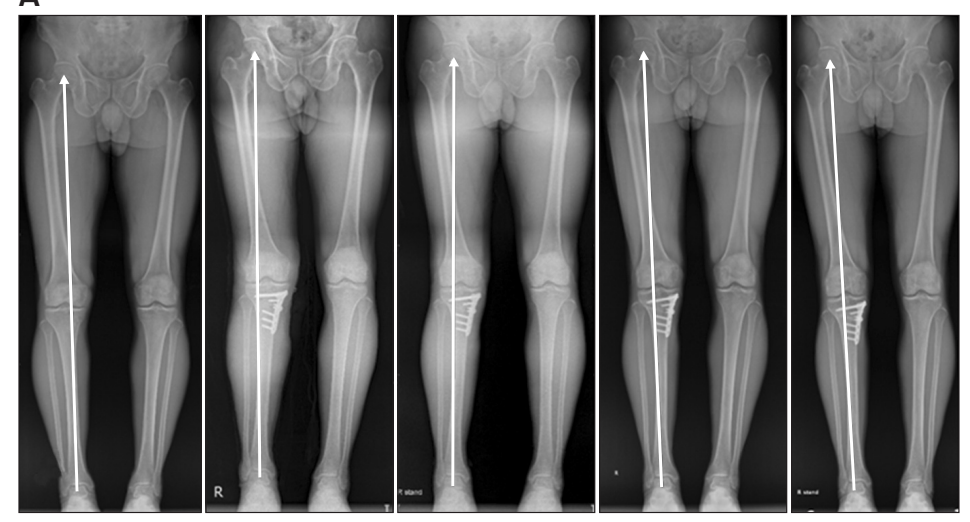

c

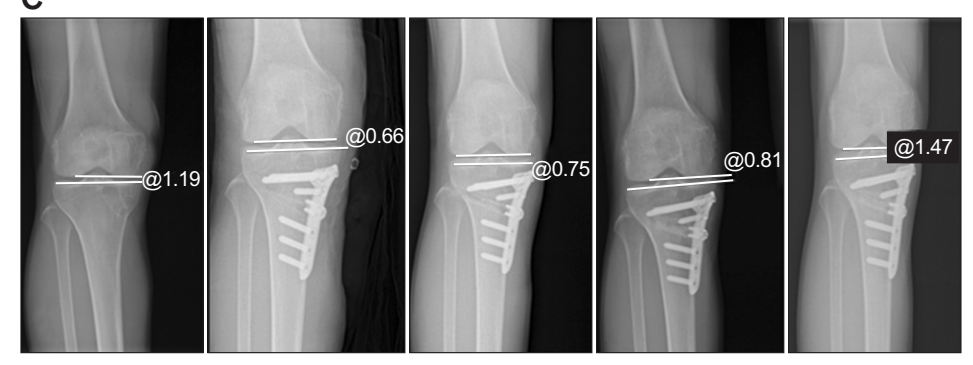

E

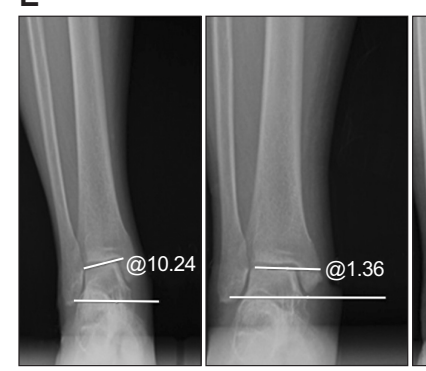

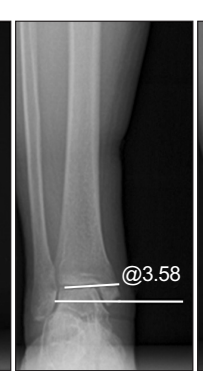

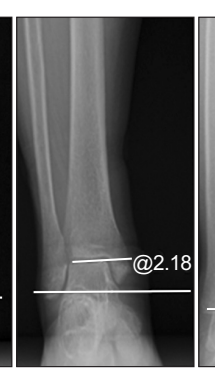

B
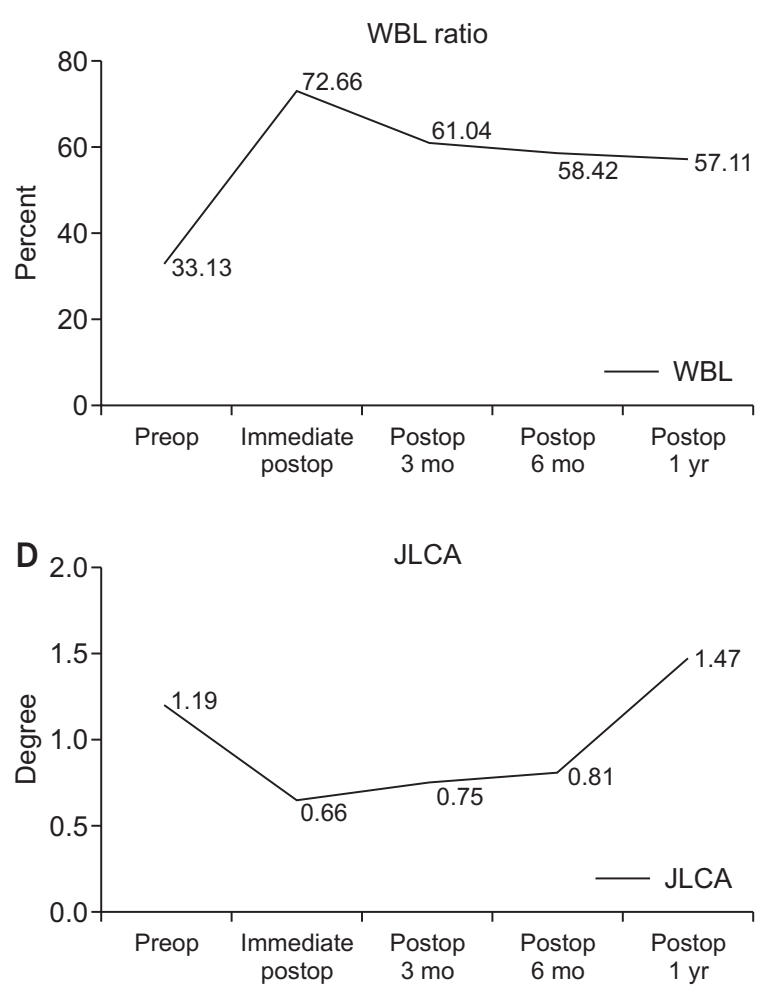

F

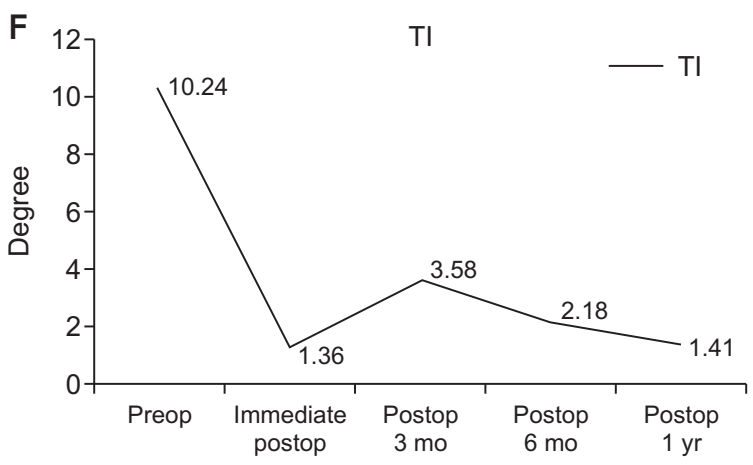

Fig. 5. Serial changes of each parameter in the recurrence group. (A) Weight bearing line (WBL) in the whole leg radiograph. (B) WBL in the polygonal line graph. (C) Joint line convergence angle (JLCA) in the whole leg radiograph. (D) JLCA in the polygonal line graph. (E) Talar inclination (TI) in the whole leg radiograph. (F) TI in the polygonal line graph. Preop: preoperative, Postop: postoperative. 
Table 2. Difference of the Parameters between the Recurrence (R) Group and No Recurrence (NR) Group

\begin{tabular}{lcrr}
\hline \multicolumn{1}{c}{ Parameter } & R group & NR group & p-value \\
\hline Postoperative 1 year WBL $(\%)$ & $37.30 \pm 10.51$ & $61.02 \pm 7.59$ & $<0.05$ \\
Immediate JLCA $\left(^{\circ}\right)$ & $2.55 \pm 1.49$ & $1.38 \pm 1.38$ & $<0.05$ \\
D-talar inclination $\left({ }^{\circ}\right)$ & $5.57 \pm 4.16$ & $3.77 \pm 2.07$ & 0.05 \\
D-distal tibia articular angle $\left({ }^{\circ}\right)$ & $4.53 \pm 2.64$ & $2.94 \pm 2.16$ & 0.18 \\
\hline
\end{tabular}

Values are presented as mean \pm standard deviation.

WBL: weight bearing line, JLCA: joint line convergence angle, D: difference=postoperative 1 year-immediate postoperative values .

\section{Discussion}

The principal findings of this study were (1) the preoperative WBL ratio showed significantly negative correlations with the serial changes of the JLCA, TI angle, and DTAA, (2) the incidence in the R group after OWHTO was 17 of 64 (26\%) at postoperative 1 year, and (3) the JLCA, TI angle, and DTAA showed more varus alignment in the $\mathrm{R}$ group than in the NR group at postoperative 6 weeks and the difference persisted up to 1 year after surgery.

Several studies have reported the correlation between HTO and coronal alignment. Lee et al. ${ }^{10)}$ compared the JLCA among patients divided into three different groups after HTO: the undercorrection group (WBL ratio, $<57 \%$ ), over-correction group (WBL ratio, $>67 \%$ ), and acceptable correction group (WBL ratio, $57 \%-67 \%)$. The JLCA was measured before and after the surgery and compared between the R and NR groups. The pre- to postoperative difference in JLCA showed a stronger correlation than preoperative JLCA, pre- to postoperative differences of the MA, and WBL ratio. However, there was a correlation between the JLCA and WBL ratio, and the JLCA of the R group was significantly lower than that of the NR group. JLCA reportedly ranges between $0^{\circ}-2^{\circ}$ in normal persons ${ }^{13)}$. In our series, more frequent $\mathrm{R}$ was observed in patients with JLCA exceeding 2 degrees. The result could imply that JLCA after correction may be a predictive factor of $\mathrm{R}$.

Changes of the ankle joint after OWHTO have been studied. The ankle joint has a longer moment arm than the knee joint. Therefore, changes could be more prominent in the ankle joint than in the knee joint ${ }^{4,11)}$. However, deformities of the hip, knee, and ankle joints all play a role in determining the overall lower extremity alignment and it seems likely that some compensatory changes occur at each joint in response to deformity at the others. In addition, these changes could be different with time, as was evident in the present study. Therefore, it is necessary to evaluate the relation between joints and serial changes.

In this study, the WBL ratio at 6 weeks postoperatively was significantly lower in the R group than in the NR group. The insufficient correction of WBL ratio lasted up to 1 year after surgery. Additionally, the insufficient correction of the JLCA of the R group at postoperative 6 weeks also lasted up to 1 year after surgery. The present findings indicate that surgeons should strive to obtain sufficient correction of WBL ratio, although the target WBL ratio of OWHTO could be acceptable from 55\% to $65 \%$ according to the patient's condition. In terms of JLCA, it is difficult to confirm whether the JLCA has been sufficiently corrected during the surgery. However, sufficient correction of JLCA would be possible with a sufficient release of the contracted medial structures including full release of the medial collateral ligament in the first part of the OWHTO. Additionally, the pre- and intraoperative values of JLCA could be compared roughly with the C-arm image by applying axial and valgus forces to the knee joint during OWHTO. Although the parameters of the ankle joint such as TI and DTAA also showed significant correlation with the preoperative WBL at the serial follow-up, surgeons cannot apply surgical intervention to adjust the parameters measured in the ankle joint while performing OWHTO. Therefore, JLCA and WBL ratio are more important as the modifiable factors during OWHTO.

Regarding the parameters related to the coronal alignment, it can be suggested that the change of MPTA could be closely related to the $\mathrm{R}$ of the varus deformity. However, in this study, we excluded the cases with collapsed MPTA within postoperative 1year due to a metal failure or a lateral hinge fracture; we only included the cases with well-maintained MPTA after postoperative 1 year. This was because it was so intuitively obvious that the collapsed MPTA would correlate with the $\mathrm{R}$ of the varus deformity. This study was intended to explain why the coronal alignment changed despite the well-maintained bony correction; therefore, $\mathrm{R}$ of the varus deformity followed by the collapsed MPTA was beyond the scope of this study.

There are some study limitations that should be considered. First, changes were only evaluated in the knee and ankle joints although hip, subtalar, and foot deformity could also affect the overall alignment. In addition, deformity of the spine would also affect the alignment. However, it has been reported that alignment of the tibial plafond and hindfoot is variable, and talar tilt is regarded as the most important factor for good clinical results and treatment of osteoarthritis ${ }^{14,15)}$. In addition, there is a limit to check the spine and hip alignment separately. Second, the evaluation only involved standing X-ray. Dynamic kinematic evaluation may provide a higher degree of accuracy compared with the 
static measurement used in this study ${ }^{9)}$. Third, serial assessment was only performed at postoperative 1 year. However, the basis of this evaluation is that serial alignment plateaued after 1 year in our previous study ${ }^{5}$. Forth, the causal relationship between the parameters is ambiguous. In this study, the changes in parameters such as KI, MA-TP angle, TI, and DTAA could be the cause or the result of the R after OWHTO. However, it is very difficult to verify whether these parameters are the cause or the result. Moreover, this paper is not intended to address the causal relationships between such parameters. Among those parameters, we recognized that the JLCA was an intraoperatively adjustable parameter. Finally, the serial change after OWHTO in this study was relatively larger than that in other studies. It may be attributable to some outliers showing severe R. Additionally, the immediate postoperative alignment may be inappropriate to evaluate the postoperative alignment due to the difficulty of standing with full knee extension.

\section{Conclusions}

Sufficient correction of the WBL and restoration of the JLCA during OWHTO are essential to prevention of $\mathrm{R}$ of varus deformity because they are the only intraoperatively modifiable factors among those associated with varus R after OWHTO.

\section{Conflict of Interest}

No potential conflict of interest relevant to this article was reported.

\section{References}

1. Lee DC, Byun SJ. High tibial osteotomy. Knee Surg Relat Res. 2012;24:61-9.

2. Lee YS, Kang JY, Lee MC, Oh WS, Elazab A, Song MK. Effect of the osteotomy length on the change of the posterior tibial slope with a simple distraction of the posterior gap in the uni- and biplanar open-wedge high tibial osteotomy. Arthroscopy. 2016;32:263-71.

3. Han SB, Bae JH, Lee SJ, Jung TG, Kim KH, Kwon JH, Nha KW. Biomechanical properties of a new anatomical locking metal block plate for opening wedge high tibial osteotomy: uniplane osteotomy. Knee Surg Relat Res. 2014;26:155-61.

4. Lee YS, Kim MK, Byun HW, Kim SB, Kim JG. Reliability of the imaging software in the preoperative planning of the open-wedge high tibial osteotomy. Knee Surg Sports Traumatol Arthrosc. 2015;23:846-51.

5. Lee YS, Lee BK, Kwon JH, Kim JI, Reyes FJ, Suh DW, Nha KW. Serial assessment of weight-bearing lower extremity alignment radiographs after open-wedge high tibial osteotomy. Arthroscopy. 2014;30:319-25.

6. Lobenhoffer P, Agneskirchner J, Zoch W. Open valgus alignment osteotomy of the proximal tibia with fixation by medial plate fixator. Orthopade. 2004;33:153-60.

7. Staubli AE, De Simoni C, Babst R, Lobenhoffer P. TomoFix: a new LCP-concept for open wedge osteotomy of the medial proximal tibia: early results in 92 cases. Injury. 2003;34 Suppl 2:B55-62.

8. Takeuchi R, Saito T, Koshino T. Clinical results of a valgus high tibial osteotomy for the treatment of osteoarthritis of the knee and the ipsilateral ankle. Knee. 2008;15:196-200.

9. Norton AA, Callaghan JJ, Amendola A, Phisitkul P, Wongsak S, Liu SS, Fruehling-Wall C. Correlation of knee and hindfoot deformities in advanced knee OA: compensatory hindfoot alignment and where it occurs. Clin Orthop Relat Res. 2015;473:166-74.

10. Lee DH, Park SC, Park HJ, Han SB. Effect of soft tissue laxity of the knee joint on limb alignment correction in openwedge high tibial osteotomy. Knee Surg Sports Traumatol Arthrosc. 2016;24:3704-12.

11. Choi GW, Yang JH, Park JH, Yun HH, Lee YI, Chae JE, Yoon JR. Changes in coronal alignment of the ankle joint after high tibial osteotomy. Knee Surg Sports Traumatol Arthrosc. 2017;25:838-45.

12. Lee YS, Lee SB, Oh WS, Kwon YE, Lee BK. Changes in patellofemoral alignment do not cause clinical impact after openwedge high tibial osteotomy. Knee Surg Sports Traumatol Arthrosc. 2016;24:129-33.

13. Chao EY, Neluheni EV, Hsu RW, Paley D. Biomechanics of malalignment. Orthop Clin North Am. 1994;25:379-86.

14. Lee WC, Moon JS, Lee HS, Lee K. Alignment of ankle and hindfoot in early stage ankle osteoarthritis. Foot Ankle Int. 2011;32:693-9.

15. Lee WC, Moon JS, Lee K, Byun WJ, Lee SH. Indications for supramalleolar osteotomy in patients with ankle osteoarthritis and varus deformity. J Bone Joint Surg Am. 2011;93:12438. 